\title{
Review and Analysis of Telework Perspective in the Administrative Systems
}

\author{
Hamed Kord ${ }^{1}$, Yousef Noushiravani ${ }^{2 *}$, Mohammad Davoud Bahadori ${ }^{3}$, Mosayeb Jahantigh ${ }^{4}$ \\ ${ }^{1}$ Faculty Member, Department of Management, University of Velayat, Iranshahr, IRAN \\ ${ }^{2}$ Faculty Member of Zabol University of Medical Sciences, IRAN \\ ${ }^{3}$ Faculty Member, Department of Computer, University of Velayat, Iranshahr, IRAN \\ ${ }^{4}$ Master of Science in Information Technology Management, University of Sistan and Baluchestan, IRAN
}

*Corresponding Author: y.n9032@yahoo.com

Citation: Kord, H., Noushiravani, Y., Bahadori, M. D. and Jahantigh, M. (2017). Review and Analysis of Telework Perspective in the Administrative Systems. Dutch Journal of Finance and Management, 1(2), 44. https://doi.org/10.29333/djfm/5820

Published: June 30, 2017

\begin{abstract}
Current world is full of change and transformation. In the corporate world, these changes are more intense and intelligent measures to respond to these changes are inevitable. Basis of majority of the organizational change is growth and impressive technology, communications and information technology. In the present era the information technology directly affects all levels of the human individual and social life is broad and deep. One of the areas of change is how people work in organizations. Severe changes in crowd composition of the workforce and the increasing entry of women into organizations that contribute to the balance between work and family life, in addition to damages such as air pollution, high consumption of fuel and energy, traffic etc. led to a growing interest organizations and communities, has been created a phenomenon called of "telework". In this paper we try to present a theoretical framework, studied the process of expanding, requirements and challenge of teleworking phenomenon and suggestion the executive solutions for its correct application in the organization.
\end{abstract}

Keywords: organization, information technology, telework

\section{INTRODUCTION}

Throughout history three pillars of human life are work, live and recreation. The method of human life and traditional entertainment are essential change with the introduction of ICT and its applications. Human is looking in cyberspace to do many of their daily activities, such as business, communication, education, and even entertainment. Mechanism cyberspace every day based on quantitative and qualitative expansion of new technologies, hardware, software, communication and mass communication increase human knowledge and the present age has created many changes in people's lives. Change with the development of new technologies, has led to more human and more efficient use of the potential of cyberspace and new ways to replace the traditional methods. Change in traditional working remotely, from home or from anywhere else, such as telework centres, a clear example of the shift in traditional working methods based on the development of the Internet, wide area networks at global, national, local and access tools such as computers and mobile phones have evolved (Jalali, 2010). When the man was able to recruit and hire people to work, and work willingly and unwillingly subject that he made his mind to. The first document in the history of recorded work and there include actions and measures by the slaves captured in battle for their lives took place. Our actions in the contemporary world or the things we do that we have to return it receives that provides our lives and livelihoods. When do we speak our minds the impression that closes the picture of production. Means activities which may lead to outcomes that can measure 
and touch; this can be located anywhere, including a factory, agricultural land, an office, a restaurant, a school, a hospital or in the street and place. Employees in these venues also have specific names. For example, names such as farmers polish workers, office workers, teachers, doctors, nurses, and so forth. Common feature of all these jobs is that they work out a product or service. This phenomenon is a manifestation of locations and job titles related as professional staff. The concept of telework provided in the early 70 and with the intensification of the phenomenon of air pollution in some industrial cities, and the oil crisis. At that time it was thought that the problem of population density in big cities can be recognized teleworking centres and encouraging people to work from home, be resolved. When Niels gave the definition of teleworking, so far it is considered as the treatment of many diseases of this organization, or a strategy used to reduce costs. Also, in response to customers' needs and balance the work-families and help to individuals with disabilities for employment (Fonner and Rollof, 2010).

\section{Theoretical Framework of Telework}

History and Definition: Today, in the new millennium with the meanings of the words used are faced with a different meaning to the term is used in the twentieth century. However, our language, our thinking and our behaviour are not consistent with these changes and we are still the traditional way we think and act; but it is expected that this conceptual change, we will immediately lead to a change in this area (Vega, 2003). Interest in telework in response to a number of conditions, and changes flared, it can be seen that the oil crisis in 1973 (a big jump in oil prices) decline in manufacturing employment, rising number of people with jobs, news, downsizing and reengineering of organizations in the 1980s and new thinking about the patterns of life and work cited. Throughout this period, the computer and telecommunications and information processing capabilities that are considered foreground, climbed while costs came down. Thus it became possible to telework (Walls, 2004). In 1987, Francis had the Francis Kinsman more than 20 years of experience in the subject of "the social, political, economic and technology and its impact on work and the art of management" lecture and book titled "teleworkers" was published. This is the first book in English to predict Teleworking and the "flexible job" is discussed. He indicated that a lot of people rather than material gain, are worried about their current life. He believes that teleworking will attract people because of the flexibility, autonomy, job satisfaction and low mobility, the potential benefits. Teleworking is not a job but a way of organizing work is built around an information processing, individuals or groups of people, away from the employer, a customer or partner, they do. Involves the use of various electronic equipment and product (outcome) is transmitted from the remote.

Advantage of Telework: Telework, despite the limitations, advantages are numerous. But what is important is that the benefits of telework from one person to another, from one organization to another, a kind of telework in another way, for different ages and even for men and women are different. Because of this, the author has examined a particular aspect of it. For example, Reed (1995), Stanworth and Stanworth (1991) the benefits such as improved quality of life, improving individual skills, flexibility in labour savings (cost, time and thought), better education increase productivity, pay based on performance rather than paying by mail, and can provide better service and more customer-centric organizations have noted. Some people also believe that as a green activity can not overlook the environmental impacts of telework (Sohrabi and Rezayian, 2006). The more benefits the individual, organizational and societal telework will be followed: 


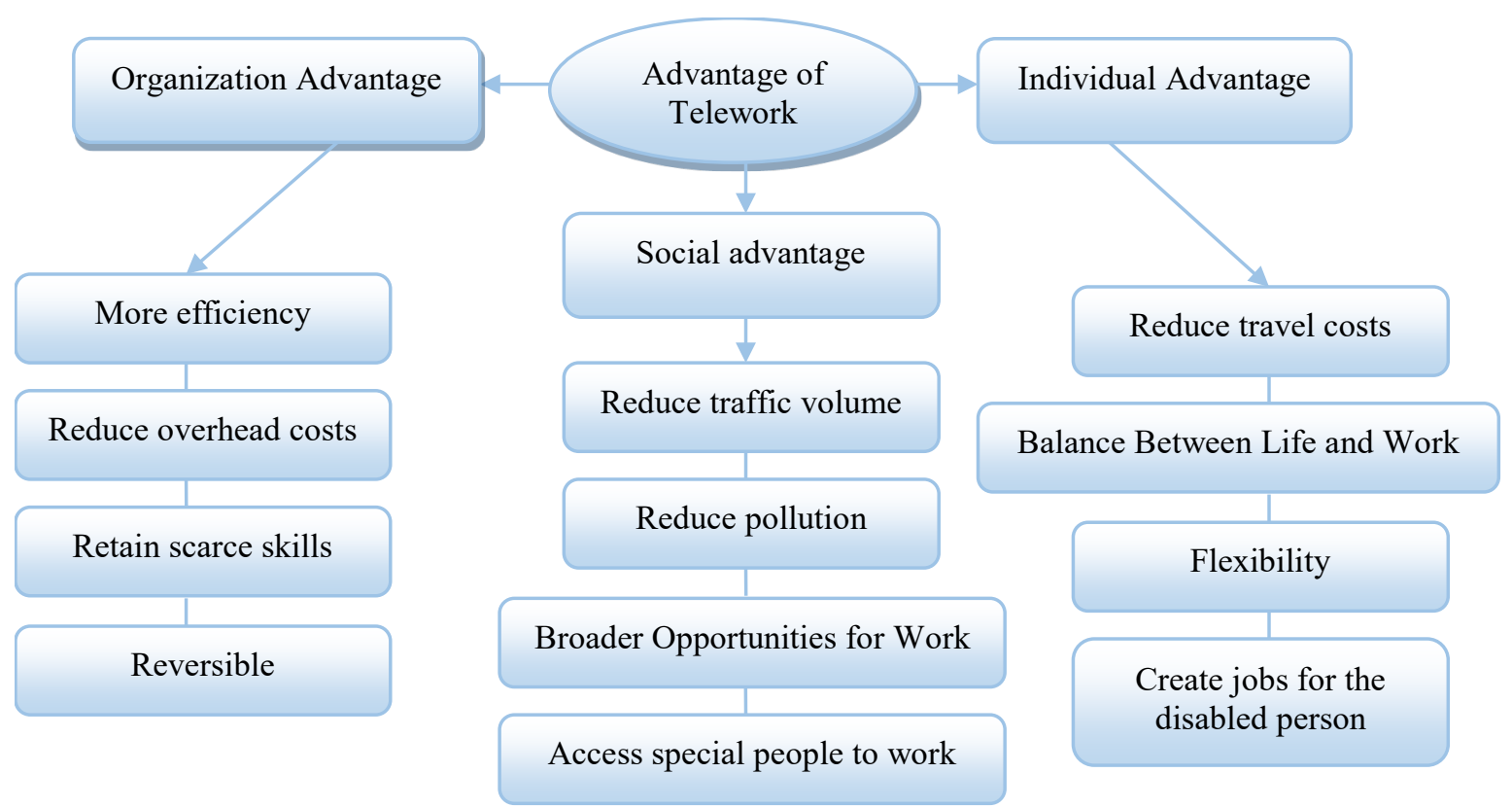

Figure 1. Advantages of Telework

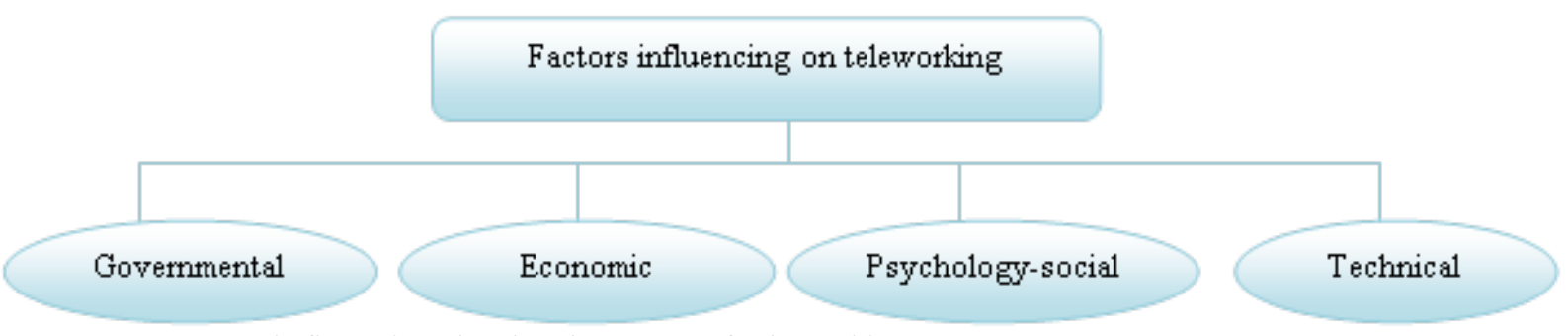

Figure 2. Factors influencing the development of teleworking

Factors influencing the development of teleworking: Most important factors showed in Figure 2.

\section{Challenges of Teleworking}

Major categories of challenges that teleworking is noteworthy that some of the challenges and problems related to the type and nature of the phenomenon of teleworking, therefore, to solve problems and deal effectively with the challenges of teleworking should be a systematic approach to the identification and their interrelationship is necessary. But looked at another way, regardless of the origins of these challenges can be divided into three general categories as follows:

Personal challenges of teleworking: Personal challenges refer to issues, problems and threats facing employee's telework and telework employees must contribute to a sense of personal and organizational or nonuse of its contribution towards solving them to action. The following are the principal challenges associated with telework. Although all these problems do not arise in all teleworking, people and organizations. Lack of proper facilities: Employee's telework usually traditional workplace facilities such as computer hardware and software more and more advanced, faster Internet connections, auxiliary equipment, suitable working space and access to such facilities as may be in the teleworking adequate.

Decreasing social relationship: work in isolation from other colleagues, and a lack of support, may lead to withdrawal and isolation employee. Professional isolation: Telework teleworking employees with loss of employment and professional relationships with colleagues are faced, for this reason, as well as that teleworkers should have sufficient expertise and the motivation to perform essential job functions.

Social isolation: The biggest challenge is teleworking employees' sense of isolation. Informal relationships in the workplace and social support, ideas and information required to perform duties effectively provides. Stress: Although one of the benefits of telework to reduce stress, working environment, but it should be noted that the poor performance of their teleworking can also cause stress levels among employees in the telework. No doubt, stress and tension increase or decrease substantially depending on the form of teleworking in the work, the nature of the family environment and the quality of the work environment.

Hard work situation: Employee's telework should be equipped with enough space for a computer and other equipment to perform their job duties in place of teleworking. Many residential facilities do not have enough to do some of teleworking. For example, even with individual motivation to perform activities that require 
concentration, when the young kids in a small apartment with noisy neighbours, or live with the problem faced. For example, the small issue of workplace employee's teleworking one of the fundamental problems in the member states of the former East Bloc.

Motivation: One of the disadvantages of teleworking, employee motivation and satisfaction, their problem is distance. Time spent in meetings with such personnel shall be exchanged information and incentives to use them. Thus, if an organization wants to take maximum advantage of employees, employees must do the work, and enjoy the feeling of being a challenge to have it. If an employee develops feelings not only of the withdrawal from service and reduced costs of recruitment and a decrease. Rather, it is ensured that employees will spend all their efforts and will determine the best possible results.

Job Satisfaction: Job satisfaction shows that person how much he loves his job, do you feel positive and a general assessment of their job or not. This evaluation consists of perceived job characteristics, and work environment is passion. Of course, in addition to environmental factors, personal characteristics and genetic factors also influence job satisfaction. Research on motivation of employees to engage in telework, shows that adaptation activities, housework and caring for children with professional work can be seen as an advantage because it increases the flexibility of working time teleworkers increased participation in sports and social activities (Konradt and Schmook, 2010). Teleworking in aspects such as reduced travel time, reduced stress, workplace, flexible work time and increase the possibility of dealing with family matters, job satisfaction is increased. However, there are some aspects such as the possibility of conflicts of work and family stress of being away from the social environment and will have a negative effect on job satisfaction. Also, if is better the quality of work life in the organization during teleworking, understandably will have a positive outcome on job satisfaction and vice versa. Concern about the lack of career advancement of individuals Teleworking individuals Teleworking always concerned about the lack of progress and improvement in the organizational hierarchy, the managers are away from the front of the eye.

\section{Organizational Challenges of Teleworking}

Some of the challenges and problems in the implementation of teleworking teleworking is the organization that administers and related organizations and although it may be based on performance and satisfaction of each employee teleworkers have different effects. However, management must make decisions and take action to resolve them. Teleworking major organizational challenges to the hearing are: Learning and knowledge sharing: teleworkers informal learning opportunities to interact with colleagues in order to develop employment, access to and transfer of tacit knowledge from their supervisor (Fonner and Rollof, 2010). Facilitate the flow of tacit knowledge in organizations through improved communication is possible. However, knowledge sharing is crucial, teleworking environment, opportunities and challenges in the process, provides. Communication and socialization of tacit knowledge creates new problems (Belanger and Allport, 2008).

Performance evaluation: Many studies suggest that improved productivity and performance of teleworkers. Although the measurement of productivity due to various factors such as size, condition and family situation is difficult teleworking, teleworking requires that managers of traditional mechanisms to monitor and evaluate performance based on the quality and appropriateness of output aside and evaluate their performance.

Information Security: information security and the protection they have always been the main affairs in organizations. If the implementations of teleworking predictions need to be made in this regard, due to security concerns and weaknesses in communication networks, ability to take risk, and even to take risks organizations have a life. Different entities in the organization and its surroundings there are the security agencies are effective. For security, the role of each of these attributes and methods to deal with them is detected. Many organizations have employees teleworking technologies are used to implement teleworking from places outside the organization. Most teleworkers to access the computational resources or specific organization, the use of remote access technology. Nature of teleworking and remote access technologies, allowing access to protected resources through external networks and external hosts that often. Because of these technologies, sources at higher risk than other technologies that are accessible only from within the organization, to give. Therefore, organizations must adopt teleworking policies specific to the security of information and communications.

Despite the necessary hardware infrastructure: Problems installing and maintaining appropriate equipment in places and situations, and lack of opportunities for communication between managers and employees are teleworking teleworking infrastructure problems. Although teleworking would reduce a lot of costs, but costs such as the costs of maintaining and updating the inevitable communication systems. Identify forces for teleworking: how to evaluate and select employees as telework, the personality and skills of Vocational and their commitment is difficult.

Costs related to the preparation and implementation of teleworking: additional financial cost of teleworking equipment at the beginning of the implementation of teleworking systems (such as computers, network systems, software packages, etc.) may enable organizations to executing the system without passion. 
Difficult access to employees during working urgency of the major advantages of working in the organization, the availability of human resources in teleworking, but it is missing or weak. Although the requirement for consideration is the availability of staff, however in emergencies, and immediate access to them impossible.

\section{Social Challenges of Teleworking}

The major social challenges of teleworking include: Definition of teleworking: As was mentioned in the definition of teleworking few examples, various scholars have offered different definitions and unique teleworking. One of the challenges of teleworking in the world is not a single definition of the term. The lack of a common definition leads to disagreement among experts, administrators, social workers and planners about the topic and examples of how to deal with this. Lack of a universally accepted definition of teleworking is causing problems.

Lifestyle: Lifestyle is one of the factors that should be considered in teleworking. Information technology will blur the line between personal and business time is. Another point that is often not considered is that teleworking should be tailored to the circumstances of life. Some families have small children at home, and for some, a work environment like home. In some cases, teleworking is a family-friendly contract. The most important factor for the success of telework, and the effective management of trust and mutual respect between employee and employer (Pyoria, 2011).

Legal challenges: teleworking agreements should be consistent with each country's labour laws are framed. Terms and conditions essential role in the regulation of relations between employers and employees who work there. Therefore, international organizations involved in occupational and employment relations in different countries as well as efforts to develop and formulate new legal regulations in this field have begun to respond to the challenges before the procedure. The main point in formulating rules and regulations for teleworking is that the new rules of solving problems related to labour relations, employment relations and conditions, capacities and potential teleworking should not interfere with the problems facing the Iranian Organization for teleworking: structural, behavioural, environmental. Identify problems and barriers to telework for organizations that want to implement these plans and mechanisms to deal intelligently with the problems and obstacles are all-important. Difficulties can be divided into three categories as follows:

(1) Behavioural problems, including:

- Lack of knowledge of the benefits and applications of remote management and general "ICT"

- Most of the weakness of foreign language

- Inefficiency and limited knowledge of Employee

(2) Structural difficulties, including:

- The high cost of hardware installation

- High cost of communication

(3) Environmental problems and obstacles, including:

- Instability and uncertainty communication lines

- Loss regulations for working remotely.

\section{Teleworking Strategies}

In general, decisions relating to macro strategies at senior management levels of an organization. Strategy is documented evidence that the combination of a basic design, research and development and has achieved Main certain reference for the decision into coming. This document is used to coordinate and organize the next steps in the matter of political definition of the word, strategy is a road map. Guidelines for the administration and governance of the community and the government of the kingdom vary, but common among them all there. Can teleworking strategies at the state level in general, consider the following three categories; each consisting of a number of related policies.

The main legislative instrument for controlling the actions of the government in the general population. The field of teleworking in particular, the government should consider all factors and proper legislation in this area to facilitate teleworking and to attract people and organizations to take steps toward it. Of such legal agreements may be noted that in formulating their requirements for reliability and flexibility for individuals to exist. In addition, the increase in mobility and job security for employees in accordance with the law which has created a sense of confidence in them. Finally, the legal requirements regarding the relationship between rights and responsibilities of each person considered the scope and responsibility of individuals to be properly cleaned and difficulties in this regard does not arise. Establishment and development of supportive institutions in order to strengthen scientific and practical subjects to participate in teleworking. Open teleworking requires major structural changes at the state level and the macro. In this regard, some smaller organizations or disappear entirely or mainly created and others has developed. The first set of institutions that are necessary to strengthen the institutions needed for teleworking infrastructures which provide the technological infrastructure. The high volume of investments and expectations 
of the infrastructure, financing and development of the institutions of government will be in line with the overall strategy. In the context of lifelong learning strategies can be adopted people.

Individuals and organizations to embrace the culture of teleworking: The development of teleworking, public knowledge and beliefs about the positive functions of that. If the legal requirements and the establishment of supportive infrastructure and institutions, but culture are not realized, teleworking will become popular and will remain only on monopoly in certain circumstances. Teleworking involves creating a culture and climate based on trust and dialogue. In addition, the creation of social security systems can be a general culture of the areas near and in accordance with the will of the people has to be willingness to teleworking.

\section{DISCUSSION AND CONCLUSION}

In recent years, statistics of women college graduates and the subsequent rise of the cortex, organizations are faced with serious challenges and new. What the community is closed to the public in mind, imagine men as breadwinners and women as housewives is. In recent years, women have increasingly entered the social and institutional spheres. To create balance between work and family roles arrangements if they do not think, that "the future consequences and negative consequences of women's organizations and community outcomes would be more positive.

One of the main consequences of this is the creation of a generation in the family will break. When both parents are forced to conduct business affairs, morning and afternoon return home, they will be forced to send their children to the centres of education, or a hobby there. The presence of children in these centres will be a longterm educational practices of the institutions with which they have grown and character commensurate with education and training others to develop methods for very little time to spend with family. Between thought and action in adolescents with family expectations profound gaps may exist that do not meet this gap will not be easy. Therefore it is necessary to assure the presence of women in society and in addition to the maintenance organization is, in the family, either qualitatively or quantitatively the presence of dodge-work them.

One of the ways to create a balance between work and life is the topic of telework. Because of its complex and multifaceted phenomenon of telework should be planned, done enough research and patience. On the other hand, because it is based on information and communication technologies, to provide infrastructure needed because of the failure to fully meet these requirements, organizations will be faced with more problems. On the other hand, the culture should be done before implementation. Because people are naturally "to resist change, resist telework phenomenon unknown because it would be much more serious. Therefore should provide the necessary training and informing people of the benefits and positive outcomes of telework, them compliance presence together is less need to strengthen and build social capital in the organization, strategies and concrete actions be pondered. In general, teleworking phenomenon that appears in the majority of organizations will be forced to implement it in your environment. So it seems to be that organizations that approach as a strategy for the future of your organization and in an era when only the study of how to implement it. Because if this option is not selected as a strategy, not enough research done on it, in the future, organizations will be forced to require the use of telework and what is clear is that in terms of voluntary choice, with focus, patience and careful doing it but in the case of coercion, confusion, chaos and confusion makes things happen with lower quality. In the end, it seems that the phenomenon of telework in the future will be inclusive, so although like any other phenomenon has negative consequences as well, studies and assessments should be sufficient to eliminate the negative consequences of the implementation is correct, accurate and scientific way be considered.

\section{REFERENCES}

Belanger, F. and Allport, D. C. (2008). Collaborative technologies in knowledge telework: an exploratory study. Info Systems J, 18, 101-121. https:// doi.org/10.1111/j.1365-2575.2007.00252.x

Fonner, L. K. and Rollof, E. M. (2010). Why Teleworkers are More Satisfied with Their Jobs than are Office Based Workers: When Less Contact is Beneficial. Journal of Applied Communication Research, 38(4), 336-361. https://doi.org/10.1080/00909882.2010.513998

Jalali, A. A. (2010). Teleworking, do's and don'ts. Age Analysts Journal, 4(40), 27.

Konradt, U. and Schmook, R. (2010). Quality of management by objectives, task related stressors, and non-task related stressors as predictors of stress and job satisfaction among Teleworkers. European Journal of Work and Organizational Psychology, 12(1), 61-79. https://doi.org/10.1080/13594320344000020

Pyoria, P. (2011). Managing telework: risks, fears and rules. Management Research Review, 34(4), 386-399. https://doi.org/10.1108/01409171111117843 
Sohrabi, B. and Rezayian, A. (2006). Telework survey of Kowsar Economic Organization. Journal of Management Message, 20(19), 173-159.

Stanworth, J. and Stanworth, C. (1991). Telework: The Human Resource Implications. London: Institute of Personel Management.

Vega, G. (2003). Managing teleworkers and telecommuting strategies, First published. United States of America: Praeger Publishers. 